\title{
Perda de primeiros molares permanentes em crianças e adolescentes atendidos na Clínica-Escola de Odontologia - UFPE
}

First permanent molar loss among children and adolescents attended at Clinic-School of Dentistry - UFPE

Pérdida de primeros molares permanentes en niños y adolescentes tratados en la ClínicaEscuela de Odontología

Alleson Jamesson da Silva ${ }^{1 *}$, Laís Cavalcanti Leal Santos ${ }^{1}$, Jessyca Maria Alencar e Sá1, Brenda Rocha Borba de Andrade ${ }^{1}$, Elizabeth Arruda Carneiro Ponzi ${ }^{1}$, Alice Kelly Barreira ${ }^{1}$.

\section{RESUMO}

Objetivo: Verificar a prevalência de perda de primeiros molares permanentes em indivíduos de 10 a 19 anos de idade atendidos em uma Clínica-Escola universitária. Métodos: Trata-se de um estudo transversal retrospectivo a partir da coleta de dados de 204 prontuários de pacientes, de ambos os sexos, com idade de 10 à 19 anos. As variáveis compreenderam aspectos relativos à perda dentária, à prevenção e fatores sociodemográficos. Para verificar as frequências e associações com as variáveis foi utilizado o teste do Quiquadrado de Pearson ou teste Exato de Fisher, quando apropriados. Resultados: Verificou-se um total de $21,57 \%$ de perda de primeiros molares permanentes, sem diferença entre sexo, local de residência, doenças sistêmicas, frequência de escovação, uso diário de fio dental e tipo de alimentação, sendo os primeiros molares permanentes inferiores os elementos mais acometidos, com 9,80\% para o 36 e $11,27 \%$ para o 46 . A associação foi positiva entre perda dentária e as variáveis idade e índice CPO-D. Conclusão: Os resultados revelam a alta prevalência de perda de primeiros molares permanentes, considerando-se a necessidade do desenvolvimento de ações preventivas e curativas afim de reduzir o número de exodontias desses elementos.

Palavras-chave: Criança, Adolescente, Perda de Dente, Dente Molar, Cárie Dentária.

\begin{abstract}
Objective: To verify the prevalence of loss of first permanent molars in children aged 10 to 19 years attending a university clinic. Methods: This is a retrospective cross-sectional study based on data collection of 204 medical records of patients of both sexes, aged 10-19 years. The variables include aspects related to tooth loss, threat and sociodemographic factors. To verify frequencies and associations with variables, Pearson's chi-square test or Fisher's exact test were used, when appropriate. Results: A total of $21.57 \%$ loss of first permanent molars was observed, with no difference between sex, place of residence, systemic diseases, frequency of brushing, daily flossing and type of feeding, being the first permanent lower molars the most affected elements, with $9.80 \%$ for 36 and $11.27 \%$ for 46 . The association was positive between tooth loss and age and CPO-D index. Conclusion: The results reveal the high prevalence of loss of first permanent molars, considering the need to develop preventive and curative actions in order to reduce the number of extractions of these elements.
\end{abstract}

Key words: Child, Adolescent, Tooth Loss, Molar, Dental Caries.

${ }^{1}$ Universidade Federal de Pernambuco (UFPE), Recife-Pernambuco. *E-mail: allesonjamesson@gmail.com SUBMETIDO EM: 9/2019 ACEITO EM: 9/2019 PUBLICADO EM: 10/2019 


\section{RESUMEN}

Objetivo: Verificar la prevalencia de pérdida de primeros molares permanentes en individuos de 10 a 19 años que asisten a una clínica universitaria. Métodos: Este es un estudio transversal retrospectivo basado en la recopilación de datos de 204 registros médicos de pacientes de ambos sexos, de 10 a 19 años. Las variables comprendieron aspectos relacionados con la pérdida de dientes, prevención y factores sociodemográficos. Para verificar las frecuencias y asociaciones con variables, se utilizó la prueba de chi-cuadrado de Pearson o la prueba exacta de Fisher, cuando fue apropiado. Resultados: Se observó una pérdida total de $21.57 \%$ de los primeros molares permanentes, sin diferencias entre sexo, lugar de residencia, enfermedades sistémicas, frecuencia de cepillado, uso diario del hilo dental y tipo de alimentación, siendo los primeros molares inferiores permanentes los elementos más afectados, con $9.80 \%$ para 36 y $11.27 \%$ para 46 . La asociación fue positiva entre la pérdida de dientes y la edad y el índice CPO-D. Conclusión: Los resultados revelan la alta prevalencia de pérdida de primeros molares permanentes, considerando la necesidad de desarrollar acciones preventivas y curativas para reducir el número de extracciones de estos elementos.

Palabras clave: Niño, Adolescente, Pérdida de Diente, Diente Molar, Caries Dental.

\section{INTRODUÇÃO}

Mundialmente, dentre as doenças infecciosas, a cárie dentária é a de ocorrência mais comum. Entre as crianças e adolescentes, $60 \%$ a $90 \%$ são acometidos por essa doença (SABER AM, et al., 2018). No Brasil, pode-se observar uma maior incidência em populações socioeconomicamente desfavorecidas (MELO FGC, et al., 2011).

Esses grupos mais desfavorecidos têm menos acesso e utilizam menos serviços preventivos e assistenciais, consomem mais sacarose, possuem piores padrões de higiene bucal e apresentam menor acesso e utilização de fluoretos, resultando em maiores danos e maiores perdas (BARBATO PR e PERES MA, 2009).

A perda dentária em uma região ou população pode ser considerada um bom indicador sobre as atitudes prevalentes em relação à saúde bucal, bem como a disponibilidade e acessibilidade dos serviços de saúde. Com a perda dos dentes, a qualidade de vida e a ingestão nutricional de uma pessoa são afetados, portanto, é essencial entender os fatores causais e contributivos para planejar e desenvolver estratégias eficazes para preveni-la (SAMUEL SS, et al., 2018).

A partir das perspectivas clínicas e de saúde pública, são necessários programas de educação em saúde bucal, direcionados principalmente a crianças de baixa idade, na tentativa de reduzir os níveis de cárie em dentes permanentes. Além disso, exames orais periódicos devem ser realizados para garantir um diagnóstico precoce de lesões cariosas e defeitos de desenvolvimento do esmalte, com atenção especial às medidas preventivas e terapêuticas precoces (LLENA C e CALABUIG E, 2018).

O primeiro molar permanente (PMP) foi relatado como o dente mais propenso à cárie na dentição permanente. Mais de $50 \%$ das crianças com mais de 11 anos têm alguma experiência de cárie neste dente, a principal causa de extração desse elemento. Entre os fatores causais para o início da doença está o longo período de tempo do desenvolvimento coronal do dente, o que o torna suscetível a defeitos cronológicos do esmalte, como a hipomineralização, um defeito qualitativo com características clínicas que variam de opacidades demarcadas branco-creme a amarelo-acastanhado, como também sua erupção por volta dos seis anos de idade (COBOURNE MT, et al., 2014; GAROT E, et al., 2017; ALKHADRA T, 2017).

A erupção dos primeiros molares permanentes na cavidade oral é calma e não apresenta sintomas, visto que estes elementos dentários não vêm a substituir dentes decíduos. Essa lerdeza e silêncio, no irromper na cavidade bucal, conduzem à uma precoce interação microorganismos-substrato (BOTELHO K, et al., 2011).

O longo período de erupção é o determinante mais influente no desenvolvimento da cárie oclusal, por meio do acúmulo de placa espessa no sistema fossa-sulco e do estágio de erupção dentária que limita a função

REAS/EJCH | Vol.11(17) | e1580 | DOI: https://doi.org/10.25248/reas.e1580.2019 Página 2 de 9 
mecânica oral. Consequentemente, lesões oclusais ativas são significativamente mais prevalentes em dentes em erupção do que em dentes totalmente irrompidos (CARVALHO JC, 2014).

Um objetivo importante dos programas de saúde bucal pediátrica é prevenir a cárie nesse elemento, pois, o mesmo desempenha um papel fundamental no desenvolvimento e crescimento das arcadas dentárias. A maioria desses programas aplica medidas específicas locais com base na vedação de fossas e fissuras, ou a aplicação de verniz fluoretado (LLENA C e CALABUIG E, 2018).

Manter os primeiros molares permanentes na cavidade oral é fundamental para o estabelecimento de uma oclusão equilibrada, pois eles constituem parte essencial do sistema estomatognático. Nesse sentido, tornase extremamente importante a realização de estudos com esta linha metodológica, a fim de aumentar o rol de dados disponíveis acerca da prevalência de perda desses elementos, possibilitando o desenvolvimento de ações que visem mudar essa realidade. Este trabalho teve como objetivo avaliar a prevalência de perdas de primeiros molares permanentes em crianças e adolescentes atendidos na Clínica-Escola de Odontologia UFPE e associar com fatores sociodemográficos e fatores relacionados à saúde bucal.

\section{MÉTODOS}

Este estudo, caracterizado como um estudo quantitativo, retrospectivo, de corte transversal, foi realizado no Curso de Odontologia da Universidade Federal de Pernambuco (UFPE), no Departamento de Clínica e Odontologia Preventiva, localizada no município do Recife, Pernambuco, Brasil. A Clínica-Escola é composta por três clínicas e um Cento de Especialidades Odontológicas, com estrutura física necessária para que a população tenha acesso a atendimento especializado em todas as áreas da Odontologia. $O$ atendimento é realizado por alunos do quinto ao décimo período da graduação, supervisionados por professores da instituição.

As informações obtidas neste estudo são confidenciais e os sujeitos da pesquisa não foram identificados quando o material de registro foi utilizado. O projeto foi submetido ao Comitê de Ética em Pesquisa com Seres Humanos do Centro de Ciências da Saúde da UFPE e aprovado com número de parecer 3.158.388.

A população do estudo foi composta por 204 prontuários de pacientes de 10 a 19 anos atendidos na Clínica Integral II - Atenção ao Adolescente e Clínica de Cirurgia Buco-Maxilo-Facial, no período entre os meses de fevereiro de 2018 e maio de 2019. A escolha dessa faixa etária para o estudo tem como justificativa o fato de que, a partir dos 10 anos, o PMP já se encontra na cavidade bucal há quatro anos e com o ápice radicular totalmente formado. As clínicas foram escolhidas com base no atendimento à essa faixa etária, onde é possível verificar a realização de exodontias anteriormente e/ou posteriormente ao atendimento inicial.

As informações referentes à identificação das perdas dentárias foram coletadas diretamente nos prontuários de atendimento de cada indivíduo, armazenados no Núcleo de Acolhimento e Pronto Atendimento do Curso de Odontologia da Universidade Federal de Pernambuco. Foram incluídas na amostra todas as fichas clínicas de indivíduos de ambos os sexos, com idades entre 10 e 19 anos e excluídos aqueles que não estavam com o prontuário devidamente preenchido, ou seja, com ausência do registro de exame dental, onde é possível verificar as perdas dentárias, informações estas necessárias para análise no estudo.

Além dos dados referentes as perdas dentárias, também foram colhidos dados sociodemográficos, como sexo, idade, local de residência e fatores relacionados à saúde geral, como número de dentes afetados por doença cárie, frequência de escovação dentária, uso diário de fio dental, doenças sistêmicas e tipo de alimentação. Todos os dados obtidos foram tabulados em planilha do Excel e, posteriormente, submetidos à análise estatística para determinação de prevalências e possíveis associações entre as variáveis analisadas com o auxílio do software Epi-Info® versão 7.2.2.16 de 2018 (Centers for Disease Control and Prevention, Atlanta, USA). Foi realizada estatística descritiva com cálculo das médias, desvios-padrões e percentuais. Para verificar se houve associações com as variáveis foi utilizado o teste do Qui-quadrado de Pearson ou teste Exato de Fisher, quando apropriados. A significância estatística de todos os testes foi considerada como p-valor $<0,05$. 


\section{RESULTADOS}

Dentre esses, $47,73 \%$ das perdas encontravam-se no arco dentário inferior, 34,09\% no arco dentário superior e 18,18\% em ambos os arcos (Tabela 1).

Tabela 1 - Distribuição dos pacientes, segundo sexo, idade, local de residência, doença sistêmica, frequência de escovação, uso diário de fio dental, tipo de alimentação, CPO-D, perda de primeiro molar permanente (total da amostra) e por arco dentário.

\begin{tabular}{|c|c|c|c|}
\hline \multirow[t]{2}{*}{ Variáveis } & \multicolumn{3}{|c|}{ Frequência } \\
\hline & $\mathbf{N}$ & $\mathbf{N}$ & $\%$ \\
\hline Sexo & 204 & & \\
\hline Masculino & & 95 & 46,57 \\
\hline Feminino & & 109 & 53,43 \\
\hline Idade & 204 & & \\
\hline 10-14 anos & & 106 & 51,96 \\
\hline $15-19$ anos & & 98 & 48,04 \\
\hline Local de residência & 185 & & \\
\hline Região Metropolitana do Recife & & 167 & 90.27 \\
\hline Outros municípios & & 18 & 9,73 \\
\hline Doença sistêmica & 200 & & \\
\hline Sim & & 24 & 12,00 \\
\hline Não & & 176 & 88,00 \\
\hline Frequência de escovação & 84 & & \\
\hline $1-2 x$ ao dia & & 43 & 51,19 \\
\hline$>3 x$ ao dia & & 41 & 48,81 \\
\hline Uso diário de fio dental & 82 & & \\
\hline Sim & & 20 & 24,39 \\
\hline Não & & 62 & 75,61 \\
\hline Tipo de Alimentação & 144 & & \\
\hline Não-cariogênica & & 58 & 40,28 \\
\hline Cariogênica & & 86 & 59,72 \\
\hline Experiência de cárie & 204 & & \\
\hline Sim & & 24 & 11,76 \\
\hline Não & & 180 & 88,24 \\
\hline Perda de Primeiro Molar Permanente & 204 & & \\
\hline Sim & & 44 & 21,57 \\
\hline Não & & 160 & 78,43 \\
\hline Arco dentário & 44 & & \\
\hline Superior & & 15 & 34,09 \\
\hline Inferior & & 21 & 47,73 \\
\hline Ambos & & 8 & 18,18 \\
\hline
\end{tabular}

Fonte: Silva AJ, Santos LCL, Sá JMA, Andrade BRB, Ponzi EAC, Barreira AK, 2019. 
Verificou-se que $21,57 \%$ dos pacientes da amostra apresentaram perda de PMP. Dos 204 pacientes da amostra, 51,96\% correspondiam a faixa etária de 10 a 14 anos e 48,04\% entre 15 e 19 anos. Em relação ao sexo, mais da metade $(53,43 \%)$ eram do sexo feminino e a maioria era residente da Região Metropolitana do Recife $(90,27 \%)$. A análise de perda de PMP por elemento, mostrou que houve $8,33 \%$ de perdas do elemento $16,4,90 \%$ de perdas do elemento $26,9,80 \%$ de perdas do elemento 36 e $11,27 \%$ de perdas do elemento 46 . Os índices CPO-D, C, P e O, respectivamente, apresentaram médias de 5,9, 3,5, 0,6 e 2,0 e desvio padrão de 5,4, 4,3, 1,3 e 2,5. Em relação as variáveis sexo, local de residência, doença sistêmica, frequência de escovação, uso diário de fio dental e alimentação, não se encontrou associação estatisticamente significativa quando associadas a perda de PMP. Entretanto, associações positivas entre perda de primeiro molar permanente com idade e índice CPO-D foram verificadas (Tabela 2).

Tabela 2 - Associação entre as variáveis perda dentária e sexo, idade, local de residência, doença sistêmica, frequência de escovação, uso diário de fio dental, tipo de alimentação e CPO-D.

\begin{tabular}{|c|c|c|c|c|c|c|c|}
\hline \multirow{3}{*}{ Variáveis } & \multicolumn{6}{|c|}{ Perda Dentária } & \multirow[b]{3}{*}{$p$-valor } \\
\hline & \multicolumn{2}{|c|}{ Total } & \multicolumn{2}{|c|}{ Sim } & \multicolumn{2}{|c|}{ Não } & \\
\hline & $\mathbf{n}$ & $\%$ & $\mathbf{n}$ & $\%$ & $\mathbf{N}$ & $\%$ & \\
\hline \multicolumn{8}{|l|}{ Sexo } \\
\hline Masculino & 95 & 100 & 16 & 16,84 & 79 & 83,16 & \\
\hline Feminino & 109 & 100 & 28 & 25,69 & 81 & 74,31 & 0,1 \\
\hline \multicolumn{8}{|l|}{ Idade } \\
\hline $10-14$ & 106 & 100 & 12 & 11,32 & 94 & 88,68 & \\
\hline $15-19$ & 98 & 100 & 32 & 32,65 & 66 & 67,35 & 0,0004 \\
\hline \multicolumn{8}{|l|}{ Local de residência } \\
\hline Região Metropolitana do Recife & 167 & 100 & 38 & 22,75 & 129 & 77,25 & \\
\hline Outros municípios & 18 & 100 & 6 & 33,33 & 12 & 66,67 & 0,4 \\
\hline \multicolumn{8}{|l|}{ Doença sistêmica } \\
\hline Sim & 24 & 100 & 6 & 25,00 & 18 & 75,00 & \\
\hline Não & 176 & 100 & 36 & 20,45 & 140 & 79,55 & 0,8 \\
\hline \multicolumn{8}{|l|}{ Frequência de escovação } \\
\hline $1-2 x$ ao dia & 43 & 100 & 8 & 18,60 & 35 & 81,40 & \\
\hline$>3 x$ ao dia & 41 & 100 & 8 & 19,51 & 33 & 80,49 & 1,0 \\
\hline \multicolumn{8}{|l|}{ Uso diário de fio dental } \\
\hline $\operatorname{Sim}$ & 20 & 100 & 4 & 20,00 & 16 & 80,00 & \\
\hline Não & 62 & 100 & 11 & 17,74 & 51 & 82,26 & 1,0 \\
\hline \multicolumn{8}{|l|}{ Tipo de alimentação } \\
\hline Não-cariogênica & 58 & 100 & 12 & 20,69 & 46 & 79,31 & \\
\hline Cariogênica & 86 & 100 & 20 & 23,26 & 66 & 76,74 & 0,8 \\
\hline \multicolumn{8}{|l|}{ CPO-D } \\
\hline Sem experiência de cárie & 24 & 100 & 0 & 0 & 24 & 100 & \\
\hline Alguma experiência de cárie & 180 & 100 & 44 & 24,44 & 136 & 75,56 & 0,01 \\
\hline
\end{tabular}

Fonte: Silva AJ, Santos LCL, Sá JMA, Andrade BRB, Ponzi EAC, Barreira AK, 2019. 


\section{DISCUSSÃO}

O presente trabalho verificou um percentual de $21,57 \%$ de perda de primeiros molares permanentes em indivíduos de 10 a 19 anos de idade atendidos em uma Clínica-Escola universitária. Em estudo realizado com crianças e adolescentes de escolas da rede pública de Campina Grande - PB, avaliando todos os molares permanentes, a prevalência foi de 17,2\% (MELO FGC, et al, 2011). Enquanto os resultados encontrados por Botelho K, et al. (2011) em uma pesquisa realizada também na Clínica-Escola da UFPE no ano de 2002, com crianças com idades entre 6 e 8 anos, a perda de primeiros molares permanentes foi de $8,2 \%$.

Esses resultados ocorrem pelo fato do primeiro molar permanente ser o dente permanente mais propenso à cárie e também suscetível a defeitos de desenvolvimento, como a hipomineralização. Por causa dessas duas condições, o comprometimento dos primeiros molares permanentes é frequentemente significativo, resultando em difícil restauração dos dentes e prognóstico questionável a longo prazo, aumentando a possibilidade de exodontia desses elementos (TEO TKY, et al., 2016).

No presente estudo, o arco dentário inferior foi o mais acometido por perdas de primeiros molares permanentes (47,73\%), resultado que corrobora com os achados de Melo FGC, et al. (2011), Coser MC, et al. (2005) e Vieira SCM e Aronita R (2003). Os motivos da maior prevalência de cárie encontrada em primeiros molares permanentes inferiores ainda não foram demostrados, no entanto, a cronologia da erupção coloca os elementos inferiores expostos ao desafio cariogênico mais precocemente e portanto, por um período de tempo maior (SULZLER KE, et al., 2018).

A localização central no arco, no sentido ântero-posterior, que os primeiros molares permanentes ocupam, é o motivo pelo qual a ausência dos mesmos é tão prejudicial para a integridade funcional do arco, causando uma deficiência de mais de $80 \mathrm{~mm}$ de superfície de mastigatória eficiente (BOTELHO K, et al., 2011).

Ao se analisar a perda dentária, segundo o elemento dentário, observou-se maior número de exodontias do elemento 46 (11,27\%), seguido do elemento $36(9,80 \%), 16(8,33 \%)$ e $26(4,90 \%)$. Estes números concordam com os resultados de Melo FGC, et al. (2011), Botelho K, et al. (2011), Barbato PR e Peres MA (2009) e Coser MC, et al. (2005). Essa realidade é consequência das grandes lesões cariosas que estes dentes podem apresentar, muitas vezes com apenas evidências mínimas de um defeito pré-existente no esmalte. O momento da erupção do primeiro molar também torna esses dentes vulneráveis à cárie dentária. Embora a experiência de cárie tenha caído continuamente na dentição permanente das crianças, os dados mais recentes demonstram que cerca de um terço dos jovens ainda têm experiência de cárie em dentina em pelo menos um desses dentes. $\mathrm{E}$, ainda atualmente, a maioria dos primeiros molares permanentes é extraída por causa da cárie dentária (COBOURNE MT, et al., 2014).

Em relação ao sexo, observou-se um percentual de perdas maior para o sexo feminino $(25,69 \%)$ em relação ao sexo masculino (16,84\%), corroborando com os estudos de Peres MA, et al. (2013); no entanto, em discordância com os resultados de Llena C e Calabuig E (2018), que encontraram maior percentual para o sexo masculino. Ao associar a perda dentária com esta variável, resultados estatisticamente significativos não foram encontrados, assim como nos estudos de Melo FGC, et al. (2011) e Samuel SS, et al. (2018).

Estudos com adultos mostram que as mulheres costumam buscar mais os serviços de saúde e consequentemente são submetidas a mais tratamentos, enquanto os homens, demonstram menos autocuidado com a saúde (GOMES R, et al., 2007; LAWDER JAC, et al., 2008).

Quando se analisou a idade, um maior número de pacientes da amostra encontrava-se na faixa etária entre 10 e 14 anos (51,96\%). Ao associar com a perda dentária, constatou-se maior perda dentária com o aumento da idade, com diferença estatisticamente significante $(p=0,004)$. Esses dados vão de encontro aos encontrados por Melo FGC, et al. (2011) e Teo TKY, et al. (2016), que encontraram maiores prevalências aos 12 e 9 anos de idade, respectivamente. É importante enfatizar que, na maioria dos indivíduos da faixa etária desse estudo, esses dentes já passaram por alguma experiência de cárie, iniciando o ciclo restaurador. Todavia, dentes restaurados inúmeras vezes, ocasionalmente podem ser extraídos, o que pode levar a alterações na oclusão se os espaços não forem reabilitados. 
A maioria dos pacientes da amostra eram residentes da Região Metropolitana do Recife $(90,27 \%)$. A perda dentária em uma região selecionada ou população nos dá uma visão sobre as atitudes prevalentes em relação à saúde bucal, bem como a disponibilidade e acessibilidade dos serviços de saúde. É essencial entender os fatores causais e contributivos, para planejar e desenvolver estratégias eficazes para reduzir a perda dentária. Quanto menor renda e escolaridade, maiores as perdas. Indivíduos mais pobres e menos escolarizados residem em localidades com menores coberturas de fluoretação de águas e de serviços odontológicos, consomem mais açúcar e escovam menos frequentemente seus dentes. Todos esses fatores contribuem para aumento da prevalência e extensão da cárie dentária e, consequentemente, das perdas dela resultantes. (SAMUEL SS, et al. 2018; PERES MA, et al. 2013).

Neste estudo, ao se analisar a frequência de escovação, observou-se que $51,19 \%$ dos pacientes escovavam os dentes de 1 a 2 vezes ao dia e 48,81\% escovavam mais de 3 vezes, diferente dos resultados obtidos por Ghanim AM, et al. (2012) onde $75,5 \%$ dos 823 indivíduos estudados escovavam de 1 a 2 vezes ao dia, e por Silva E, et al. (2018) onde $33,3 \%$ dos 390 adolescentes da amostra escovavam com a mesma frequência. Já no estudo de Davoglio RS, et al. (2009) realizado com 1.170 adolescentes, $77,8 \%$ destes realizavam escovação com uma frequência de, pelo menos, 3 vezes ao dia.

Quando se observou o hábito de uso diário de fio dental, verificou-se que o mesmo se apresentou ausente em $75,61 \%$ dos adolescentes da amostra, número este elevado em comparação com os $46,7 \%$ obtidos no estudo supracitado, realizado por Silva E, et al. (2018). Em uma pesquisa de Freddo SL, et al. (2008) com 1.171 escolares, apenas $31,9 \%$ faziam uso diário de fio dental.

Existe uma forte associação entre a frequência de escovação e a presença de cárie, sem grandes variações na razão de chances comparando a escovação duas ou mais vezes ao dia versus menos de duas vezes ao dia, ou uma ou mais vezes ao dia contra menos de uma vez por dia. No entanto, a maioria das pessoas, e particularmente as crianças, são incapazes de garantir o controle adequado do biofilme através da escovação sozinha. A este respeito, o uso de um creme dental fluoretado durante a escovação é possivelmente o fator mais importante na prevenção da cárie (LLENA C e CALABUIG E, 2018).

Em se tratando do tipo de alimentação, é evidente que as perdas dentárias estão constantemente associadas a uma dieta rica em açúcar. Nesse contexto, observa-se no presente estudo uma alta frequência de indivíduos com dieta cariogênica, correspondendo a 59,72\%. Reforçando essa afirmativa, percebeu-se que todos os pacientes que apresentaram alguma perda dentária, possuíam esse tipo de alimentação. Todavia, ao procurar associações entre a presença de perdas de primeiros molares permanentes e as variáveis relacionadas ao uso diário de fio dental, tipo de alimentação e doenças sistêmicas, resultados estatisticamente significativos $(p<0,05)$ não foram encontrados, reforçando os resultados de Melo FGC, et al. (2011) e Samuel SS, et al. (2018). Coloca-se a necessidade de mais pesquisas, com amostras maiores e desenho controlado a fim de elucidar essa questão.

Na população estudada, $88,24 \%$ dos pacientes apresentaram alguma experiência de cárie ao longo da vida. Ao associar a perda de primeiro molar permanente e o índice CPO-D, verificou-se resultados estatisticamente significativos $(p=0,01)$. Entre os indivíduos da amostra do presente estudo, um só paciente necessitou realizar exodontia de todos os primeiros molares permanentes.

Ao traçar as médias dos índices C, P, O e CPO-D, encontrou-se números relativamente altos quando comparados com os resultados do SB Brasil 2010. Essa diferença pode ser explicada pelo tipo de pesquisa realizada em uma unidade de atendimento, onde pacientes com necessidade de tratamento são os mais frequentes, diferente da amostra de um estudo populacional. Quando analisadas em conjunto, a média do índice CPO-D foi de 5,99, resultados estes superiores à média encontrada pelo SB Brasil 2010, onde, segundo o Ministério da Saúde (2012), verificou-se que as crianças brasileiras na faixa etária de 12 anos de idade e adolescentes entre 15 e 19 anos expressaram, respectivamente, em média, os índices de 2,07 e 4,25 elementos dentários com alguma experiência da doença cárie. Os menores índices se dão nas regiões Sudeste e Sul para essas idades, em contrapartida, nas regiões Norte, Nordeste e Centro-Oeste médias mais altas foram obtidas. 
O presente trabalho apresenta limitações relacionadas à utilização de dados secundários e ao tipo de estudo transversal, o qual não permite inferir causalidade. No entanto, apresenta dados importantes quanto à perda do primeiro molar permanente em uma população tão jovem, destacando a necessidade do desenvolvimento de políticas de promoção de saúde e de ampliação de acesso ao tratamento odontológico a fim de preservar esses elementos dentários, possivelmente por toda a vida do indivíduo.

\section{CONCLUSÃO}

A prevalência de perda de primeiros molares permanentes observada neste estudo foi considerada elevada quando analisada a faixa etária associada ao público alvo atendido, sendo os primeiros molares permanentes inferiores mais acometidos, em maior proporção o elemento 46. A perda dentária foi associada a idade e ao índice CPO-D, mas não foi influenciada pelo sexo, local de residência, doença sistêmica, frequência de escovação, uso diário de fio dental e tipo de alimentação.

\section{REFERÊNCIAS}

1. ALKHADRA T. A systematic review of the consequences of early extraction of first permanent first molar in different mixed dentition stages. Journal of International Society of Preventive and Community Dentistry, 2017; 7(5): 223-226.

2. BARBATO PR, PERES MA. Perdas dentárias em adolescentes brasileiros e fatores associados: estudo de base populacional. Revista de Saúde Pública, 2009; 43(1): 13-25.

3. BOTELHO K, et al. Condição clínica dos primeiros molares permanentes: de crianças entre 6 e 8 anos de idade. Odontologia Clínico-Científica, 2011; 10(2): 167-171.

4. BRASIL. Ministério da Saúde. Secretaria de Atenção à Saúde. Secretaria de Vigilância em Saúde. SB Brasil 2010: Pesquisa Nacional de Saúde Bucal: resultados principais. Brasília: Ministério da Saúde; 2012; 116 p.

5. CARVALHO JC. Caries process on occlusal surfaces: evolving evidence and understanding. Caries Research, 2014; 48(4): 339-346.

6. COBOURNE MT, WILLIAMS A, HARRISON M. National clinical guidelines for the extraction of first permanent molars in children. British Dental Journal, 2014; 217(11): 643-648.

7. COSER MC, et al. Frequência de cárie e perda de primeiros molares permanentes. Revista Gaúcha de Odontologia, 2005; 53(1): 1-84.

8. DAVOGLIO RS, et al. Fatores associados a hábitos de saúde bucal e utilização de serviços odontológicos entre adolescentes. Cadernos de Saúde Pública, 2009; 25(3): 665-667.

9. FREDDO SL, et al. Hábitos de higiene bucal e utilização de serviços odontológicos em escolares de uma cidade da Região Sul do Brasil. Cadernos de Saúde Pública, 2008; 24(9): 1991-2000.

10. GAROT E, et al. Analytical evidence of enamel hypomineralisation on permanent and primary molars amongst past populations. Scientific Reports, 2017; 7(1712): 1-10.

11. GHANIM AM, et al. Trends of oral health care and dental treatment needs in relation to molar incisor hypomineralisation defects: a study amongst a group of Iraqi schoolchildren. European Archives of Paediatric Dentistry, 2012; 13(4): 171-178.

12. GOMES R, NASCIMENTO EF, ARAUJO FC. Por que os homens buscam menos os serviços de saúde do que as mulheres? As explicações de homens com baixa escolaridade e homens com ensino superior. Cadernos de Saúde Pública, 2007; 23(3): 565-574.

13. LAWDER JAC, et al. Conhecimento e práticas em saúde bucal entre usuários de serviços odontológicos. Pesquisa Brasileira em Odontopediatria e Clínica Integrada, 2008; 8(3): 321-326.

14. LLENA C, CALABUIG E. Risk factors associated with new caries lesions in permanent first molars in children: a 5year historical cohort follow-up study. Clinical Oral Investigations, 2018; 22(3): 1-8.

15. MELO FGC, et al. Perda precoce de molares permanentes e fatores associados em escolares de 9,12 e 15 anos da rede pública municipal de Campina Grande, Estado da Paraíba, Brasil. Acta Scientiarum Health Sciences, 2011; 33(1): 99-105.

16. PERES MA, et al. Perdas dentárias no Brasil: análise da Pesquisa Nacional de Saúde Bucal 2010. Revista de Saúde Pública, 2013; 47(3): 78-89.

17. SABER AM, et al. Consequences of early extraction of compromised first permanent molar: a systematic review. $B M C$ Oral Health, 2018; 18(59): 1-15.

18. SAMUEL SS, et al. Nature and pattern of primary teeth extractions in a tertiary care hospital setting in South India. Indian Journal of Dental Research, 2018; 29(2): 186-189. 
19. SILVA E, et al. Saúde bucal de adolescentes quilombolas e não quilombolas: um estudo dos hábitos de higiene e fatores associados. Ciência e Saúde Coletiva, 2018; 23(9): 2963-2978.

20. SULZLER KE, et al. Cronologia de Erupção do Primeiro Molar Permanente em Crianças dos Municípios de Santa Helena e Três Barras do Paraná, PR/Brasil. Revista Brasileira de Ciências da Saúde, 2018; 22(3): 189-194.

21. TEO TKY, et al. Lower first permanent molars: developing better predictors of spontaneous space closure. European Journal of Orthodontics, 2016; 38(1): 90-95.

22. VIEIRA SCM, ARONITA R. Perda de Primeiros Molares Permanentes em Escolares do Recife - Pernambuco - Brasil. Revista Brasileira de Ciências da Saúde, 2003; 7(1): 9-16. 